\title{
A safe and simple procedure for laparoscopic hepatectomy with combined diaphragmatic resection
}

\author{
Keisuke Oyama ${ }^{1}$ (1) S Shin Nakahira ${ }^{1} \cdot$ Sakae Maeda ${ }^{1} \cdot$ Akihiro Kitagawa $^{1} \cdot$ Yuki Ushimaru $^{1} \cdot$ Nobuyoshi Ohara $^{1}$. \\ Yuichiro Miyake $^{1} \cdot$ Yoichi Makari $^{1} \cdot$ Ken Nakata $^{1} \cdot$ Junya Fujita ${ }^{1}$
}

Received: 2 July 2021 / Accepted: 4 August 2021 / Published online: 11 August 2021

(c) The Author(s) 2021

\begin{abstract}
Diaphragmatic resection may be required beneath the diaphragm in some patients with liver tumors. Laparoscopic diaphragmatic resection is technically difficult to secure in the surgical field and in suturing. We report a case of successful laparoscopic hepatectomy with diaphragmatic resection. A 48-year-old man who underwent laparoscopic partial hepatectomy for liver metastasis of rectal cancer 20 months ago underwent surgery because of a new hepatic lesion that invaded the diaphragm. The patient was placed in the left hemilateral decubitus position. The liver and diaphragm attachment areas were encircled using hanging tape. Liver resection preceded diaphragmatic resection with the hanging tape in place. Two snake retractors were used to secure the surgical field for the inflow of $\mathrm{CO}_{2}$ into the pleural space after diaphragmatic resection. The defective part of the diaphragm was repaired using continuous or interrupted sutures. Both ends of the suture were tied with an absorbable suture clip without ligation. In laparoscopic liver resection with diaphragmatic resection, the range of diaphragmatic resection can be minimized by performing liver resection using the hanging method before diaphragmatic resection. The surgical field can be secured using snake retractors. Suturing with an absorbable suture clip is conveniently feasible.
\end{abstract}

Keywords Laparoscopy $\cdot$ Hepatectomy $\cdot$ Diaphragm $\cdot$ Surgical technique

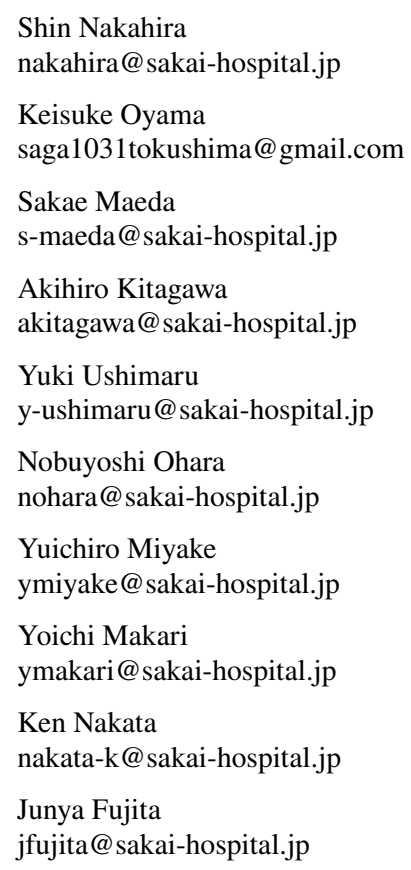

1 Department of Surgery, Sakai City Medical Center, 1-1-1 Ebarajicho, Nishi-Ku, Sakai City, Osaka 593-8304, Japan

\section{Abbreviations \\ EOB-MRI Gadolinium ethoxybenzyl diethylenetriamine pentaacetic acid-Enhanced magnetic reso- nance imaging}

\section{Introduction}

Laparoscopic liver resection has been proven to be a better alternative than open liver resection in patients with liver metastases of colorectal cancer based on perioperative and oncological outcomes [1]. Liver metastases are associated with $23 \%$ of colorectal cancers [2]. Some patients with subdiaphragmatic liver metastases of colorectal cancer require diaphragmatic resection; $3.3 \%$ of liver metastases from colorectal cancer required diaphragmatic resection [3]. It has been reported that liver resection combined with diaphragmatic resection resulted in good surgical and oncological outcomes [4]. In patients with liver metastases invading the diaphragm, diaphragmatic resection is required to obtain negative surgical margins. When laparoscopic diaphragmatic resection and suturing is performed, maintaining 
pneumoperitoneum is difficult due to the inflow of $\mathrm{CO}_{2}$ into the pleural space. Laparoscopic diaphragmatic suturing is technically difficult due to respiratory movement, narrow surgical field, and upside-down conditions. It is necessary to devise a technique to minimize the range of diaphragmatic resection and suture easily. We report a case in which laparoscopic hepatectomy with diaphragmatic resection was safely performed using a simple procedure.

\section{Case report}

A 48-year-old man underwent laparoscopic partial liver resection of segment 8 for liver metastasis of rectal cancer. Laparoscopic lower anterior resection was performed in patients with stage T3 rectal cancer. The pathological diagnosis was adenocarcinoma with no lymph node metastasis and the surgical margins of the tumor were negative. Twenty months after surgery, computed tomography revealed a new lesion beneath the diaphragm. Gadolinium ethoxybenzyl diethylenetriamine pentaacetic acid-enhanced magnetic resonance imaging (EOB-MRI) revealed a hypointense tumor located just beneath the diaphragm at the liver segment 8 on the hepatobiliary phase (Fig. 1). The surgery was performed due to growth in tumor size. The patient was placed in a left hemilateral decubitus position, and five laparoscopic ports were placed as described in Fig. 2. Pneumoperitoneum was established through the right lateral umbilical 12-mm camera port inserted using the open-entry method. Adhesions from the previous surgery were dissected. Tumor invasion to the diaphragm was detected while the right lobe was mobilized. The invaded area of the diaphragm was encircled using a hanging tape. Liver resection preceded diaphragmatic resection. Due to severe adhesions around the hepatoduodenal ligament and the small extent of resection volume, liver resection was performed without the Pringle maneuver.

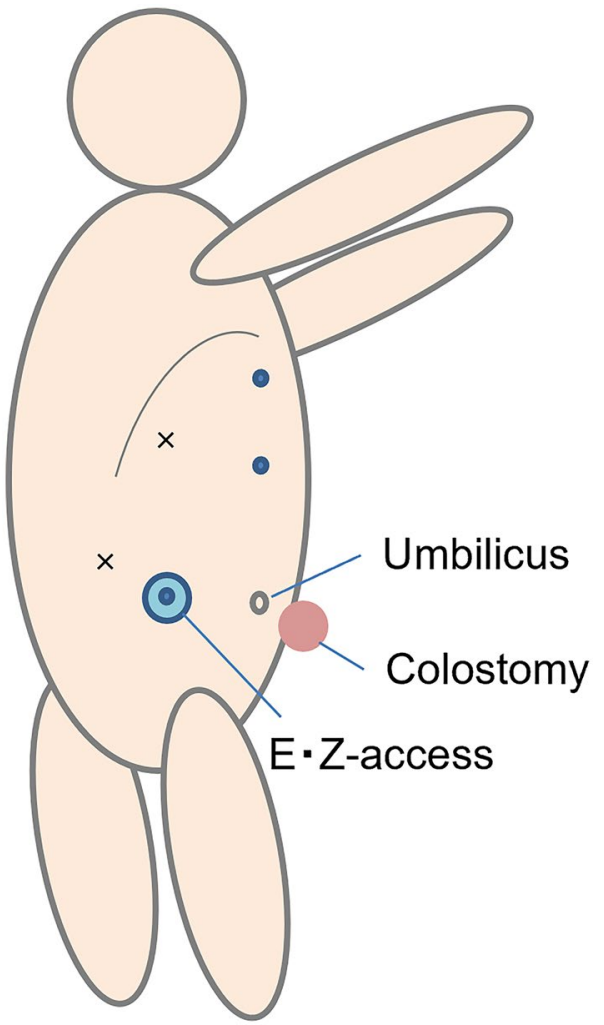

Fig. 2 The illustration of the patient and port placement. The patient was placed in the left hemilateral decubitus position. EZ-access was inserted through the right lateral umbilical. Circles represent $12-\mathrm{mm}$ and crosses represent 5-mm ports. 12-mm EZ-access, 12-mm epigastric, 12-mm upper abdominal, 5-mm right subcostal and 5-mm right lateral ports were inserted

Liver resection was performed using HARMONIC ACE® (Ethicon Endo-Surgery, Cincinnati, OH, USA) and CUSA ${ }^{\circledR}$ (Clarity Ultrasonic Surgical Aspirator System, Integra LifeSciences Corporation, NR Ireland Limited) while hanging the liver tumor with tape. The inflow of $\mathrm{CO}_{2}$ into the pleural

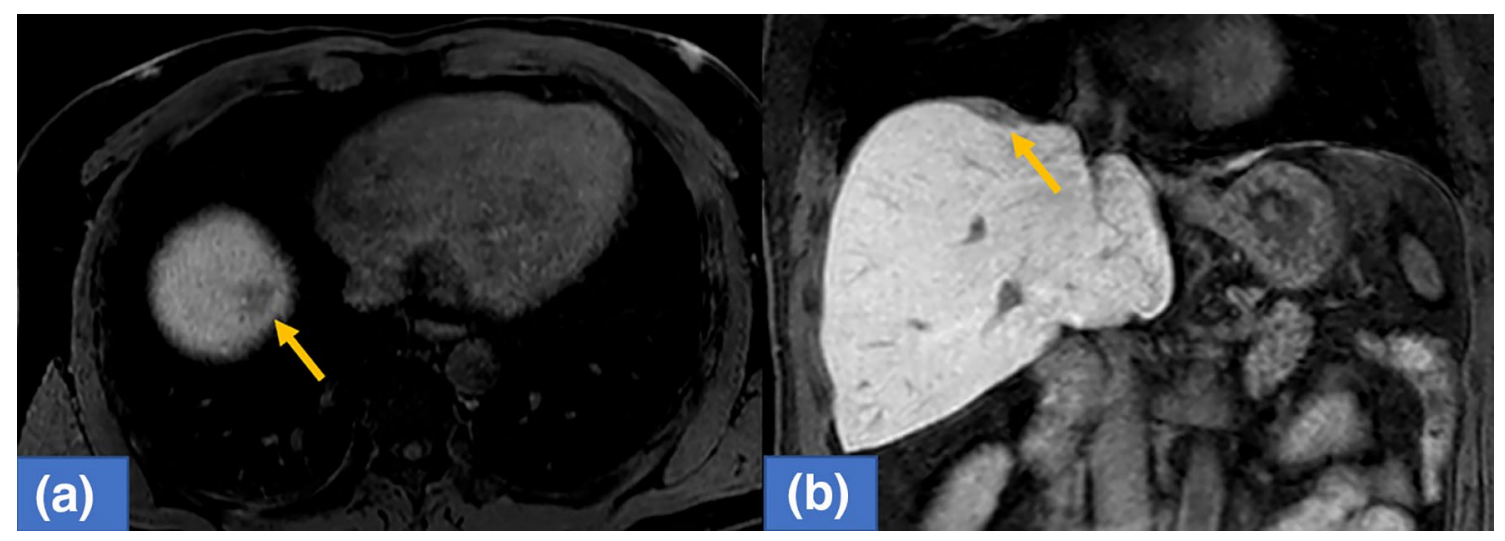

Fig. 1 EOB-MRI revealed a hypointensity tumor located just below the diaphragm at liver segment 8 on hepatobiliary phase (arrow). (a: axial sections, b: coronal sections) 
space after diaphragmatic resection created a poor surgical field due to the development of unsustainable pneumoperitoneum. The liver and diaphragm were held down using two snake retractors to secure the surgical field. The diaphragm defect was repaired with continuous or interrupted sutures placed using LAPRA-TY ${ }^{\circledR}$ (Ethicon Endo-Surgery, Cincinnati, OH, USA) clips and 3-0 Vicryl sutures. Both ends of the string were tied using a LAPRA-TY® clip without ligation. Continuous or interrupted sutures were placed based on the tension of the diaphragm while reducing the pneumoperitoneum pressure from 10 to $6 \mathrm{mmHg}$, and respiration was temporarily stopped while consulting with the anesthesiologist. The surgery was completed with a 14-French chest tube insertion. The patient had no respiratory or circulatory abnormalities during the surgery. The total operative time was $272 \mathrm{~min}$ with a $10-\mathrm{mL}$ blood loss. The image of the surgical method is shown in Fig. 3. The video of the surgery is available online only (Supporting Information). The postoperative course was uneventful, the chest tube was removed after confirming no air leakage on the first postoperative day, and the patient was discharged 8 days after the surgery. The pathological diagnosis was liver metastasis of rectal cancer invading the diaphragm. The surgical margins of the tumor were negative. The tumor weighed $5 \mathrm{~g}$, including the diaphragm. The excision range of the diaphragm was $34 \times 44 \mathrm{~mm}$ (Fig. 4). Eighteen months after the surgery, no abnormalities were found in the diaphragm regarding respiratory symptoms and imaging findings. Written informed consent was obtained from the patient to publish this video article and any accompanying images presented in this study, and patient anonymity was preserved.

\section{Discussion}

In repairing the diaphragm, primary suture or mesh placement is used based on the defect range. Reconstruction with pedunculated muscle flaps such as the external oblique muscle has also been reported [5]. Excessive tension in the diaphragm repair can lead to a risk of anastomotic leakage and adverse respiratory effects. Placing a mesh for diaphragm repair can also cause postoperative infections and pain. In laparoscopic hepatectomy with diaphragmatic resection, it is necessary to minimize the range of diaphragmatic resection. However, in the case of diaphragmatic resection before liver resection, the liver can be easily mobilized; the diaphragmatic resection cannot be performed while confirming the tumor invasion area into the diaphragm in laparoscopic hepatectomy with diaphragmatic resection. Minimal resection can be performed while confirming the range of diaphragmatic invasion by performing liver resection before diaphragmatic resection. When hepatectomy precedes

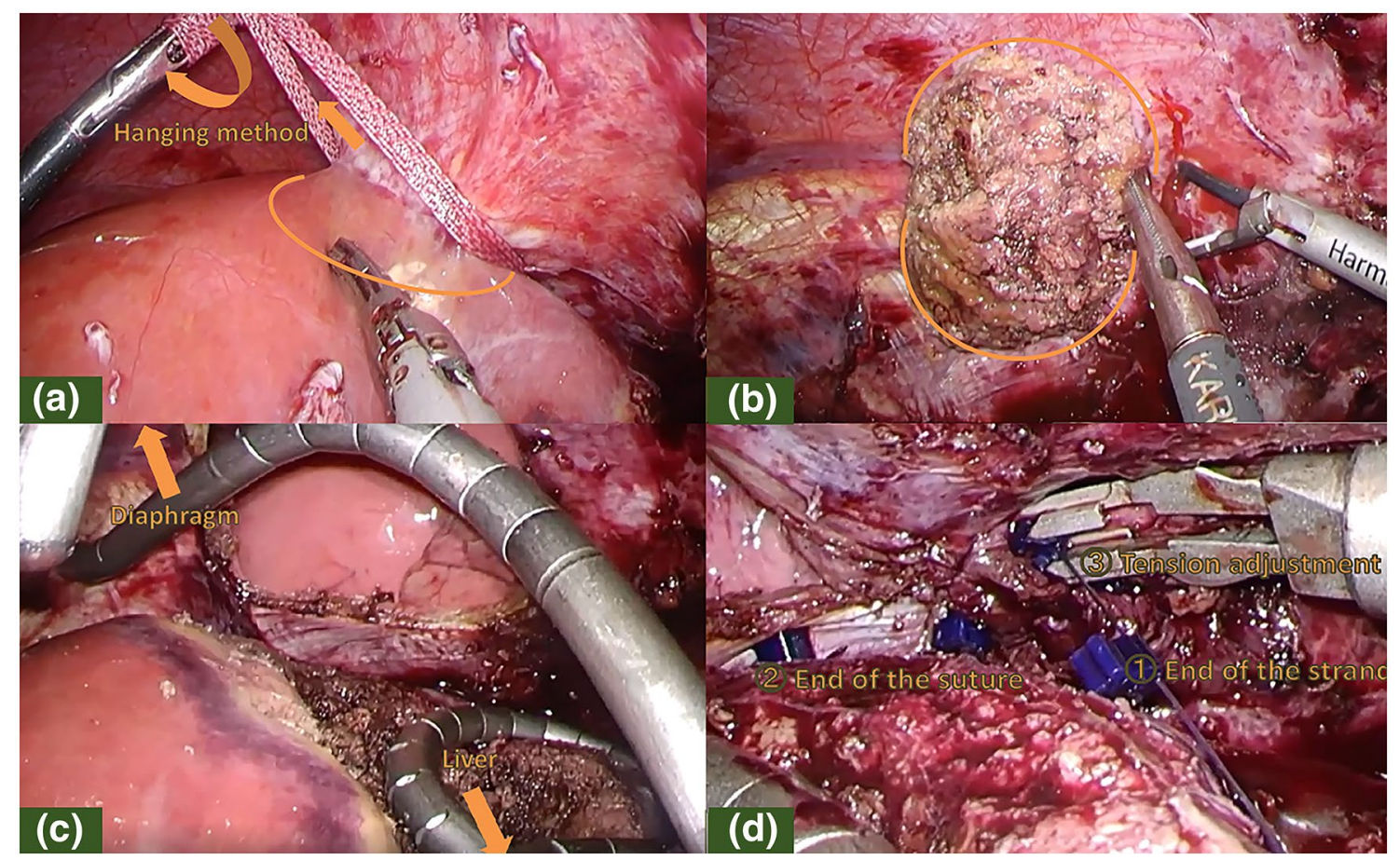

Fig. 3 Surgical image. a Liver resection using the hanging method prior to diaphragmatic resection. b Diaphragmatic resection while confirming the invading range of the tumor. c Securement the surgical field using two snake retractors. d Repairing the diaphragm with
LAPRA-TY® clips. (1)Attaching LAPRA-TY® clips to the end of the string. (2)Attaching the end of the continuous sutures. (3)Adjusted to the tension of the diaphragmatic sutures 


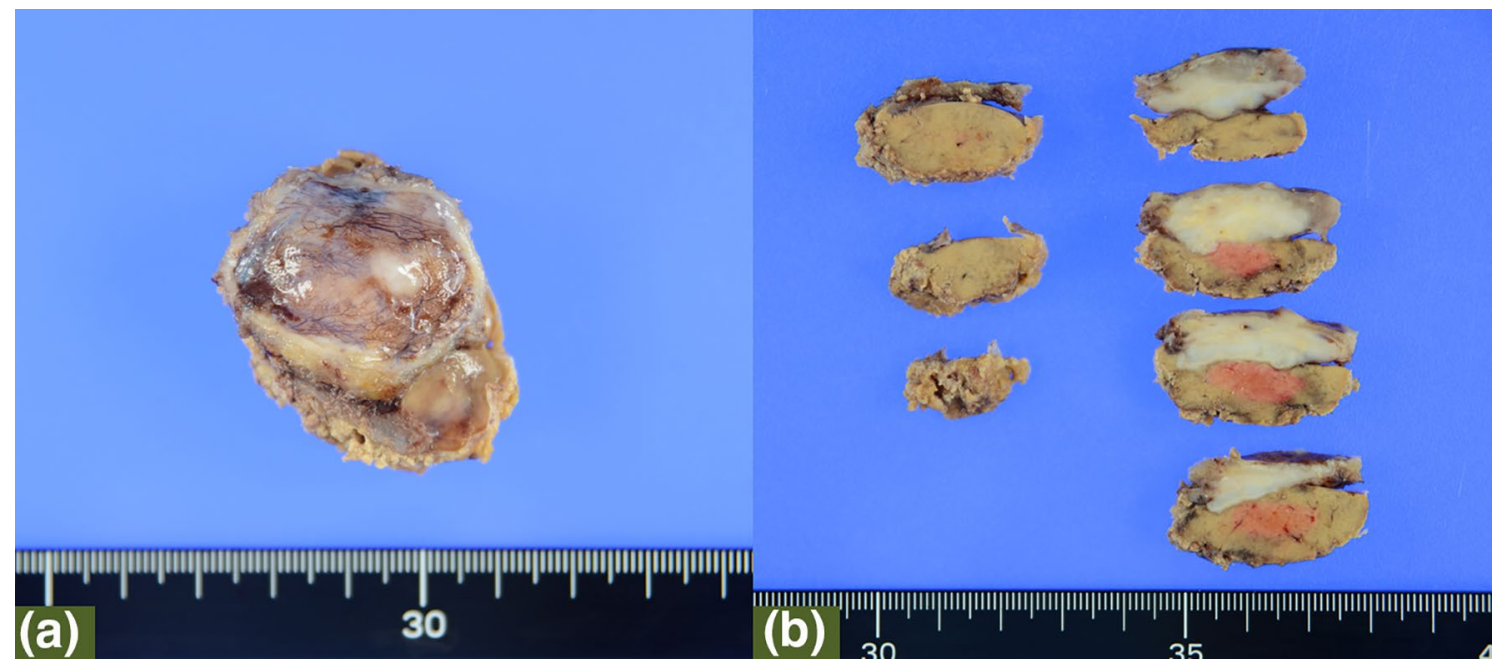

Fig. 4 a The surgical specimen. The pathological diagnosis was liver metastasis of rectal cancer. The weight of the resected liver including the diaphragm was $5 \mathrm{~g}$. The excision range of the diaphragm was $34 \times 44 \mathrm{~mm}$. b The cutting surface of specimen. The tumor had invaded the diaphragm. The surgical margins of the tumor were negative diaphragmatic resection, the mobility of the liver may be restricted because the diaphragm and liver are fixed. By encircling the invaded area of the diaphragm with tape and hanging the liver tumor, even if the liver is not mobilized freely, the hanging tape can be pulled and rolled up to control the transection plane and maintain a good tension. A thoracoscopic approach may be useful in cases of repeat hepatectomy and lesions beneath the diaphragm [6]; however, our procedure is considered superior in terms of confirming the invading range of the diaphragm and minimizing the resection range. Following diaphragmatic resection, the inflow of $\mathrm{CO}_{2}$ into the pleural space creates a poor surgical field due to the development of unsustainable pneumoperitoneum. It is necessary to devise surgical field expansion and suturing in a state where the pneumoperitoneum cannot be maintained. In previous reports, pleural air was evacuated by a transthoracic catheter or a suction tip introduced via laparoscopic trocar to secure the surgical field [4, 7]. In our case, the surgical field can be secured using snake retractors. Snake retractors can be inserted through EZ-access ${ }^{\circledR}$ (Hakko Medical Co, Nagano, Japan) and lateral 5-mm ports. The bent shape permits surgical field deployment without interference with the other forceps. The surgical field can be easily secured without any invasion into the pleural cavity using a snake retractor. It is also necessary to devise a suitable procedure for diaphragm repair due to the difficulties of laparoscopic diaphragm sutures. The diaphragm tension could reduce the pressure exerted by the pneumoperitoneum, thereby facilitating suturing [8]. Suturing with continuous or interrupted sutures using an absorbable suture clip according to the tension of the diaphragm is conveniently feasible. An absorbable suture clip can be sutured without ligation by attaching it to the end of the string. There are cases in which LAPRA-TY ${ }^{\circledR}$ clips are used in diaphragmatic injury [9], which can significantly reduce technical difficulties. Nonabsorbable sutures cannot be used when using an absorbable suture clip. Non-absorbable sutures are recommended for patients with diaphragmatic injuries and diaphragmatic hernias [10]. On the other hand, it is reported that the suture method should be selected according to the grade of diaphragmatic injury. Primary sutures with absorbable suture material can be performed if the diaphragm defect is within $10 \mathrm{~cm} \mathrm{[11,12].} \mathrm{There} \mathrm{were} \mathrm{no} \mathrm{reports} \mathrm{of} \mathrm{anastomotic} \mathrm{leak-}$ age using absorbable sutures in patients who underwent hepatectomy with diaphragmatic resection. Moreover, no short or medium-term complications were observed in our patient. If the range of diaphragmatic resection is minimized as in this procedure, no problems may arise from suturing with an absorbable suture clip and using absorbable sutures. In this case, 3-0 Vicryl was used because the diaphragmatic resection was minimized and the tension was not strong. However, in cases of high abdominal pressure such as obesity or when the resection range is close to $10 \mathrm{~cm}, 2-0$ Vicryl or thicker sutures and two-layer sutures are recommended. The 2-0 Vicryl can be attached with LAPRA-TY® clips, and the needle can be taken in and out through the EZaccess $®$. We found no reports on respiratory and circulatory abnormalities in patients who underwent laparoscopic diaphragmatic resection; however, there are existing reports on tension pneumothorax due to intraoperative diaphragmatic injury [13]. The laparoscopic surgical procedure should be stopped immediately if respiratory and circulatory abnormalities occur. Moreover, safe surgery is possible by temporarily stopping respiration when resecting or suturing the 
diaphragm. Cooperation with the anesthesiologist is especially crucial when performing laparoscopic diaphragmatic resection. We performed laparoscopic hepatectomy with diaphragmatic resection in a patient with liver metastasis of colorectal cancer, and the diaphragmatic resection was safely performed using a simple procedure.

Supplementary Information The online version contains supplementary material available at https://doi.org/10.1007/s13691-021-00506-x.

Author contributions KO drafted the manuscript. SN, SM, AK, and KO performed the surgery. All authors participated in the discussion and approved the final version of the manuscript.

Funding This work did not receive any funding.

\section{Declarations}

Ethics declarations There are no conflict of interest associated with this manuscript. The patient provided informed consent for all data written in this study and patient anonymity was preserved.

Open Access This article is licensed under a Creative Commons Attribution 4.0 International License, which permits use, sharing, adaptation, distribution and reproduction in any medium or format, as long as you give appropriate credit to the original author(s) and the source, provide a link to the Creative Commons licence, and indicate if changes were made. The images or other third party material in this article are included in the article's Creative Commons licence, unless indicated otherwise in a credit line to the material. If material is not included in the article's Creative Commons licence and your intended use is not permitted by statutory regulation or exceeds the permitted use, you will need to obtain permission directly from the copyright holder. To view a copy of this licence, visit http://creativecommons.org/licenses/by/4.0/.

\section{References}

1. Dagher I, O'Rourke N, Geller DA et al (2009) Laparoscopic major hepatectomy: an evolution in standard of care. Ann Surg 250:856-860
2. Sugihara K (2000) Liver resection for colorectal metastases. Jpn J Gastroenterol Surg 33:128-133

3. Takano S, Teshima Y, Masuda H et al (1991) Clinical study in cases of hepatectomy for liver metastasis of colo-rectal cancer. Usefulness of combined chemotherapy using a reservoir. J Jpn Soc Coloproctol 44:260-264

4. Kazaryan AM, Aghayan DL, Fretland ÅA et al (2020) Laparoscopic liver resection with simultaneous diaphragm resection. Ann Transl Med 8:214

5. Tanizaki H, Ryu M, Simamura Y et al (1994) A case of report: a repair of the right hemidiaphragm by the right external oblique muscle for a huge HCC invading the diaphragm. Jpn J Gastroenterol Surg 27:2171-2175

6. Teramoto K, Kawamura T, Takamatsu S et al (2003) Laparoscopic and thoracoscopic partial hepatectomy for hepatocellular carcinoma. World J Surg 27:1131-1136

7. Lainas P, Camerlo A, Conrad C et al (2015) Laparoscopic right hepatectomy combined with partial diaphragmatic resection for colorectal liver metastases: Is it feasible and reasonable? Surgery 158:128-134

8. Rehman J, Landman J, Kerbl K et al (2001) Laparoscopic repair of diaphragmatic defect by total intracorporeal suturing: clinical and technical considerations. Jsls 5:287-291

9. Choi J, Pan J, Forrester JD, et al (2019) LAPRA-TY for laparoscopic repair of traumatic diaphragmatic hernia without intracorporeal knot tying. Trauma surgery \& acute care open 4:e000334

10. Hanna WC, Ferri LE, Fata P et al (2008) The current status of traumatic diaphragmatic injury: lessons learned from 105 patients over 13 years. Ann Thorac Surg 85:1044-1048

11. Yasuhisa Ando KO, Murakami T, Kozai J, Suzuki H, Furuichi Y, Matsukawa H, Jun Uemura HS, Asano E, Oshima M, Usuki H, Suzuki Y (2019) A study on eight cases of traumatic diaphragmatic injury. J Abdom Emerg Med 39:815-819

12. Asensio JA, Trunkey DD, Current Therapy of Trauma and Surgical Critical Care E-Book (2008) Elsevier Health Sciences

13. Dawson R, Ferguson CJ (1997) Life-threatening tension pneumothorax during laparoscopic cholecystectomy. Surg Laparosc Endosc 7:271-272

Publisher's Note Springer Nature remains neutral with regard to jurisdictional claims in published maps and institutional affiliations. 